\title{
Die berufspolitische Heimat für alle Berufsgruppen aus Nephrologie und Dialyse Arbeitsgemeinschaft für nephrologisches Personal e.V.
}

Die Arbeitsgemeinschaft für nephrologisches Personal e. V. (AfnP) ist der mitgliederstärkste, gemeinnützige Berufsverband für nephrologisches Personal in Deutschland. Mit mehr als 1400 Mitgliedern ist die AfnP ein starker Berufsverband, der für das nephrologische Personal in nun fast 30 Jahren Verbandsgeschichte vieles erreicht hat und in der Zukunft noch vieles erreichen will.

\section{Kooperationen}

Die AfnP nimmt maßgeblich Einfluss auf die Veränderungen und Entwicklungen in der Nephrologie und Dialyse. Dies ist nur in Kooperation mit den ärztlichen Fachgesellschaften und wichtigen gesellschaftlicher Institutionen möglich. Um diese Kooperationen ist die AfnP kontinuierlich bemüht. Mit der Gesellschaft für Nephrologie (GfN), dem Arbeitskreis Transplantationspflege e. V. (AKTX) und den Psychonephrologen hat die AfnP einen Programmbeirat für die Erstellung des jährlichen Symposiums in Fulda gebildet. Ein recht herzlicher Dank geht in diesem $\mathrm{Zu}$ sammenhang an die Industrie, die uns bei der Durchführung des Symposiums unterstützt.

\section{"Die Anlaufstelle für alle ${ }^{66}$}

Wir möchten die Anlaufstelle für alle Berufsgruppen aus dem Tätigkeitsfeld Nephrologie und Dialyse sein. Wir haben uns verschiedene Ziele gesetzt:

- Förderung der Fort- und Weiterbildung

- Verbesserung der Struktur-, Prozessund Ergebnisqualität in der Versorgung und Betreuung von nierenkranken Menschen
- Erweiterung des Informationsaustausches zwischen allen beteiligten und kooperierenden Partnern

- konstruktive Kommunikation mit privaten, kommunalen und staatlichen Stellen.

\section{Eine Mitgliedschaft, die sich lohnt}

Nur gemeinsam in einem starken Verband werden wir gehört. Wir bieten unseren Mitgliedern:

- kostenlose Teilnahme am jährlichen Symposium (Nichtmitglieder zahlen 50 Euro Eintritt)

- kostenlosen Bezug der Zeitschrift „Dialyse aktuell“ (Georg Thieme Verlag KG, Stuttgart), erscheint achtmal jährlich zum Einzelpreis von 6,50 Euro

- den jährlichen Symposiumsband auf CD

- preiswerte regionale Seminare zu verschiedenen Themen

- laufende Informationen über aktuelle Themen aus der Nephrologie

- schnellen Kontakt über unsere Geschäftsstelle zu regional aktiven AfnPMitgliedern und Arbeitsgruppen.

Jahresbeitrag: 20,00 Euro
Mitglied kann jeder werden, der einer in der Nephrologie tätigen Berufsgruppe angehört. Hierzu gehören neben nephrologischen Pflegekräften beispielsweise auch Arzthelfer, Techniker und Gesundheitspfleger.

In den meisten Bundesländern haben wir Ländervertreter, die Ihnen - ebenso wie die Mitglieder des Vorstandes - für Fragen, Anregungen und Informationen (z. B. zu regionalen Fortbildungen) gerne zur Verfügung stehen.

Ihre Marion Bundschu 